\section{Dormant microbes: scouting ahead or plodding along?}

SIR — In his Essay 'Microbial awakenings' (Nature 457, 1083; 2009), Slava Epstein proposes that microbial cells stochastically revert from a state of dormancy to a growing state, allowing a clonal population of dormant cells to exploit rare and transient conditions in an environment with unpredictable periods of feast and famine. This 'scout' strategy is compatible with some - but not all explanations for the general unculturability of environmental microbes.

Other explanations include oxidative stress and substrateaccelerated death on transfer to the laboratory environment, and slow-growth strategies. If most microbes in energy-limited and spatially heterogeneous environments are slow-growing species ('K-strategists'), they may be permanently prepared for a very slow but steady existence under nutrient limitation. Rapid growth won't occur because the cells would not then be ready for the end of a brief nutrient flush. Microbes using the $K$ strategy are always prepared for resource exhaustion. As a result, they grow only slowly in laboratory culture, as noted for many 'unculturable' soil bacteria.

Other microbes, rarer in nutrient-poor environments, respond rapidly to nutrient flushes and grow at much higher rates when resources are plentiful. However, these microbes ('r-strategists') must re-enter a dormant state before such a nutrient flush is exhausted. This transition is an active and energy-requiring process, and cell death is rapid if the transition cannot be completed. Once such cells are in a dormant state, they wait for the next nutrient flush while slowly losing viability.

Therefore, Epstein's scout theory could apply to

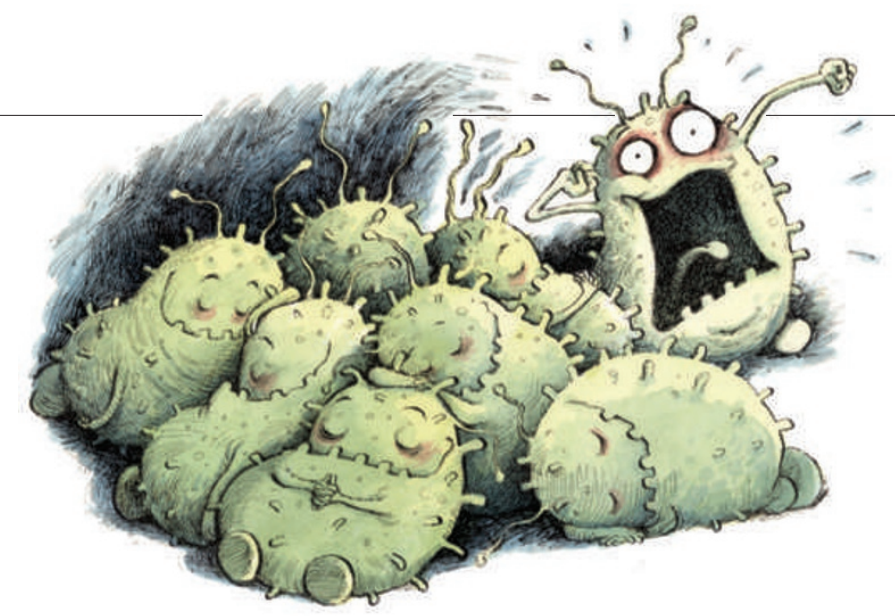

"Evolution stopped when the first caveman spoke."

Tom Wolfe, page 837

opportunistic $r$-strategists, but not so easily to $K$-strategists.

Peter H. Janssen AgResearch Limited, Grasslands Research Centre, Palmerston North 4442, New Zealand e-mail: peter.janssen@agresearch. co.nz

\section{Dormant microbes: time to revive some old ideas}

SIR - A number of researchers have already investigated, and demonstrated in detail, some of the propositions put forward in Slava Epstein's Essay 'Microbial awakenings' (Nature 457, 1083; 2009).

For instance, Epstein says "researchers could attempt to identify signalling compounds used by scouts, if they exist, and use these molecules to induce growth of dormant kin". This has been investigated by G. V. Mukamolova and colleagues (Proc. Natl Acad. Sci. USA 95, 8916-8921; 1998).

The proposition that "single cells could be cultivated, allowing them enough time for scout production unimpeded by faster growing species" has also been demonstrated (A. S. Kaprelyants et al. FEMS Microbiol. Lett. 115, 347-352; 1994). The phenomena of transient dormancy and culturability - which Epstein says "contrasts with scientific expectations" - have been studied and reviewed extensively (see, for example, G. V. Mukamolova et al. Adv. Microb.
Physiol. 47, 65-129; 2003).

Evidently ideas, as well as microbes, may remain dormant for some time.

Douglas Kell School of Chemistry and Manchester Interdisciplinary Biocentre, University of Manchester, Manchester M17DN, UK e-mail:dbk@manchester.ac.uk

The reading list for the Essay 'Microbial awakenings' (Nature 457, 1083; 2009) was published online, but Nature inadvertently omitted to refer to this list in the PDF version.

\section{Technology takes on deadlines for fetal human rights}

SIR - The view expressed in Patricia Pranke and João Carlos Silveiro's Correspondence 'Human rights cannot cover cells that were never in the womb' (Nature 458,$147 ; 2009$ ) is one of the standard components in a belief system that detaches personhood from its genetic basis. (Others include birth, viability, ability to feel pain, and various developmental milestones.)

The issue is clouded by our prejudices about what looks human and by the way fetal development seems to be a continuum. This belief system is weakened by its arbitrariness, which is being exposed as technology pushes back the boundaries. The implantation argument will become irrelevant when 'artificial wombs' make ectogenesis possible. There is no reason to expect this will not be developed in the future, whether governments try to prohibit it or not - as has been predicted for many years (Canadian Royal Commission on New Reproductive Technologies Proceed With Care; Canadian Gov. Publ., 1993).

Michael C. WendI Department of Genetics, Washington University Medical School, Box 8501, St Louis, Missouri 63108, USA e-mail:mwendl@wustl.edu

\section{More acclaim for Darwin's theory of sexual selection}

$\mathrm{SIR}$ - In this bicentennial year of Charles Darwin's birth, it is gratifying to see Nature devoting a wealth of comment to the great man and to evolution. But like much other scholarly perspective, your emphasis is on natural selection, the first of Darwin's great ideas.

Yet his second great thesis, on sexual selection, is in some ways his greatest work, because it is far less obvious than natural selection. After all, Alfred Russel Wallace also discovered natural selection, but no one else envisaged anything like sexual selection, which, even after Darwin's exposé, remained something of an anathema. Sexual selection is responsible for some of the most spectacular behaviours and characters in nature (picture the peacock's tail), but it was largely ignored during the modern synthesis and only really became mainstream in the 1970s.

Since then, we have come to realize the importance of sexual selection for much of biology, from driving rapid molecular evolution to speciation, the subject of Darwin's best-known work, On the Origin of Species. In this celebratory year, we need also to acknowledge the importance of this historically maligned mechanism of evolution.

David J. Hosken School of Biosciences, University of Exeter, Cornwall Campus, Penryn, Cornwall TR10 9EZ, UK d.j.hosken@exeter.ac.uk 